\title{
COMPARAÇÃO ENTRE METODOLOGIAS PARA AVALIAR A IDADE À PRIMEIRA CONCEPÇÃO EM ÉGUAS PURO-SANGUE INGLÊS
}

\author{
COMPARISON AMONG METHODOLOGIES FOREVALUATION OF AGE ATFIRST \\ CONCEPTION IN THOROUGHBRED MARES
}

\author{
Mota, M.D.S. ${ }^{*}$, Taveira, R. ${ }^{1}$ e Oliveira, H. ${ }^{1}$ \\ 1'Departamento de Melhoramento e Nutrição Animal.FMVZ.UNESP. Botucatu. Brasil. *mdsmota@fca.unesp.br
}

\section{Palavras chave adicionais}

Dados censurados. Equino. Reprodução.

\section{RESUMO}

No Brasil, devido à existência do ano hípico para a raça Puro-Sangue Inglês, os dados reprodutivos são geralmente truncados, já que os criadores tentam obter produtos nascidos no começo do ano hípico, a fim de aproveitarem a vantagem competitiva (animais mais desenvolvidos, maduros e treinados) destes em relação aos animais nascidos mais tardiamente no mesmo ano hípico. Para análise destes dados deve-se utilizar metodologias adequadas. Sendo assim, este trabalho objetivou comparar três metodologias: método da máxima verossimilhança restrita, com a utilização do aplicativo MTDFREML, análise bayesiana sem dados censurados por meio do programa MTGSAM e análise bayesiana com dados censurados pelo programa LMCD, para avaliação da idade à primeira concepção em éguas da raça Puro-Sangue Inglês, a fim de verificar seu impacto na escolha de reprodutores durante a seleção. $O$ arquivo de dados continha 3509 registros de idade à primeira concepção (meses) de éguas da raça Puro-Sangue Inglês. As estimativas de herdabilidades obtidas foram 0,23 , 0,30 e 0,0926 (escala log), para o MTDF, MTGSAM e LMCD, respectivamente. Considerando todos os animais do pedigree (6713), as correlações de ranking variaram de 0,91 a 0,99 . Quando considerado apenas os garanhões (656), variaram de 0,48 a 0,99 (considerando diferentes porcentagens de machos selecionados) entre as metodologias de avaliação. As maiores mudanças na classificação geral foram observadas quando comparado o LMCD com as outras duas metodologias. Considerando que o modelo linear

\section{AdDitiOnAL KEYWORDS}

Censured data. Equine. Reproduction.

censurado é o mais adequado para análise de características com dados censurados, observouse que as informações de censura levariam a escolha de diferentes animais durante o processo de seleção, quando comparado com as outras duas metodologias de análise.

\section{SUMMARY}

In Brazil, due to the breeding season for Thoroughbred, the reproductive data are normally truncate, since the breeders try to get animals that were born at the beginning of the breeding season in order to take their competitive advantages (more developed, mature and trained animals) compared to animals born later in the same breeding season. To analyze these data suitable methods should be used. Then, this paper aims to compare three methodologies: the method of maximum restricted likelihood, using MTDFREML, bayesian analysis without censured data by software MTGSAM and bayesian analysis with censured data by software LMCD, to evaluate age at first conception in thoroughbred mares, in order to verify its impact on the choice of stallions during selection. The database contained 3509 records for age at first conception (months) for thoroughbred mares. The heritability estimates were $0.23,0.30$ and 0.0926 (log scale), for MTDF, MTGSAM and LMCD, respectively. Considering all animals in the pedigree (6713), ranking correlations varied from 0.91 to 0.99 . When only stallions were considered (656), those varied from 0.48 to 0.99 (considering different percentages of selected males) between evalua- 


\section{IDADE À PRIMEIRA CONCEPÇÃO EM ÉGUAS PURO-SANGUE INGLÊS}

tion methods. The highest changes in the general classification were observed when LMCD was compared to the other two methods. As the linear censured model is the most suitable for trait analysis with censured data, it was observed that censure information would lead to the choice of different animals during the selection process, when compared to the two other methodologies.

\section{INTRODUÇÃO}

Os equinos, ao longo do processo evolutivo, desenvolveram estratégias reprodutivas que garantissem que suas progênies nascessem em época apropriada do ano, associada ao aumento do fotoperíodo, temperatura e disponibilidade de alimentos (Cilek, 2009).

O conhecimento dos índices reprodutivos na criação de cavalos é de extrema importância, uma vez que possibilita avaliar o estágio de eficiência em que se encontra a atividade, diagnosticar pontos de estrangulamento e projetar melhorias tecnológicas, além de nortear esquemas de seleção dentro dos rebanhos (Taveira et al., 2001). A precocidade sexual dos animais é um aspecto de extrema importância a ser alcançado dentro dos planos de criação de um haras, já que diminui o efetivo inativo do rebanho, proporcionando melhor retorno econômico. Existem diferentes maneiras de se estudar a precocidade reprodutiva nos animais domésticos, normalmente incluindo idades à primeira cobertura, primeiro parto e primeira concepção, todas elas de certa forma dependentes do desejo do criador. Neste sentido, a idade à primeira concepção apresenta-se como característica reprodutiva que vem despertando interesse de profissionais e criadores de diferentes espécies como medida de precocidade sexual de fêmeas. Embora ainda haja pouco interesse no setor eqüino com relação a características desta natureza (excetuandose criações de cavalos destinados ao abate), a idade à primeira concepção pode auxiliar programas de seleção que visem melhorar o desempenho dos animais.
Para a maioria das características analisadas nas avaliações genéticas utilizamse modelos lineares, em que se supõe distribuição normal, tanto dos valores genéticos, como do resíduo. Neste sentido, o método da máxima verossimilhança restrita (REML) vem sendo comumente utilizado para a obtenção de estimativas dos componentes de variância e covariância necessários para a estimação dos parâmetros genéticos. Entretanto, tem sido observado diversas vezes que a presença de animais com valores discrepantes para algumas características faz com que a distribuição de determinadas características não se enquadre adequadamente na forma da distribuição normal (Korsgaard et al., 1999). Assim, a análise bayesiana aparece como alternativa de grande flexibilidade, tanto em relação aos modelos que podem ser utilizados nas análises (Gianola, 2000), quanto em relação às inferências que podem ser realizadas a partir dos resultados (Blasco, 2001).

Além disso, no Brasil, devido à existência do ano hípico para a raça Puro-Sangue Inglês (espaço de 12 meses compreendido entre 10 de julho e 31 de junho), os dados reprodutivos são geralmente truncados, já que os criadores tentam obter produtos nascidos no começo do ano hípico, a fim de aproveitarem a vantagem competitiva (animais mais desenvolvidos, maduros e treinados) destes em relação aos animais nascidos mais tardiamente no mesmo ano hípico (Cunningham, 1991).

Embora os modelos lineares comumente utilizados não tratem de maneira adequada as informações geradas por este tipo de manejo, podendo mascarar os resultados das análises, são largamente utilizados nas avaliações genéticas devido a sua fácil interpretação. Assim, alguns modelos lineares têm sido propostos para características que apresentam informações censuradas (Korsgaard et al., 1999; Sorensen et al., 1998). Estas informações ocorrem quando apenas parte da vida produtiva do animal é conhecida. $\mathrm{O}$ fato de não se considerar os 
dados censurados em análises estatísticas pode provocar severa distorção das inferências (Sorensen et al., 1998).

Com base no exposto, este trabalho teve como objetivo a comparação de três metodologias, método da máxima verossimilhança restrita, com utilização do aplicativo MTDFREML, análise bayesiana sem dados censurados por meio do programa MTGSAM e análise bayesiana com dados censurados pelo programa LMCD, para avaliação da idade à primeira concepção em éguas da raça Puro-Sangue Inglês, a fim de verificar seu impacto na escolha de reprodutores durante o processo de seleção.

\section{MATERIALEMÉTODOS}

Os dados utilizados neste estudo foram fornecidos pelo stud book da Associação Brasileira dos Criadores do Cavalo de Corrida (ABCCC), estando contidos no arquivo de pedigree do CD-ROM desenvolvido por esta entidade em 1999. Avaliaram-se informações provenientes de 3509 éguas da raça Puro-Sangue Inglês, nascidas entre 1970 e 1993, cuja genealogia aprofundou-se até a terceira geração, totalizando 6713 animais na matriz de parentesco.
O modelo utilizado para as três metodologias avaliadas neste estudo (método da máxima verossimilhança restrita e análise bayesiana por meio dos programas MTGSAM e LMCD) incluiu, além do efeito aleatório do animal, o efeito fixo de grupo de contemporâneo (510), formado por éguas do mesmo criador (1036), nascidas no mesmo ano (1970 a 1993) e época (1-julho; 2-agosto; 3-setembro; 4-outubro; 5-novembro; 6dezembro; 7-janeiro a junho), e com no mínimo três animais cada (tabela I).

Em termos matriciais tem-se:

$$
\mathrm{Y}=\mathrm{X} \beta+\mathrm{Za}+\varepsilon
$$

em que:

$\mathrm{Y}=$ é o vetor das observações para IPCON (escala logarítmica em se tratando do LMCD);

$X=$ é a matriz de incidência dos efeitos fixos;

$\beta=$ é o vetor dos efeitos fixos;

$Z$ = é a matriz de incidência dos efeitos genéticos diretos;

$\mathrm{a}=$ é o vetor dos efeitos genéticos diretos;

$\varepsilon=$ é $\mathrm{o}$ vetor dos erros aleatórios associados às observações.

A idade à primeira concepção para as éguas que pariram machos, fêmeas, natimorto

Tabela I. Distribuição do número de éguas de acordo com ano e mês de nascimento. (Distribution of number of mares according to year and month of birth).

\begin{tabular}{lccclc}
\hline Nascimento & № éguas & Nascimento & № éguas & Mês de nascimento & № de éguas \\
\hline 1970 & 107 & 1982 & 143 & Janeiro - Junho & 33 \\
1971 & 108 & 1983 & 139 & Julho & 709 \\
1972 & 115 & 1984 & 141 & Agosto & 846 \\
1973 & 122 & 1985 & 147 & Setembro & 807 \\
1974 & 116 & 1986 & 145 & Outubro & 687 \\
1975 & 129 & 1987 & 151 & Novembro & 319 \\
1976 & 133 & 1988 & 160 & Dezembro & \\
1977 & 138 & 1989 & 173 & & \\
1978 & 136 & 1990 & 175 & & \\
1979 & 141 & 1991 & 187 & & \\
1980 & 140 & 1992 & 194 & & \\
1981 & 137 & 1993 & 232 & & \\
\hline
\end{tabular}


e que abortaram foi calculada pela subtração da data final da primeira cobertura por sua data de nascimento. As informações dos animais cujas ocorrências após a data final da primeira cobertura foram vazia, sem notícia e serviu em outra raça, utilizou-se a data final da segunda cobertura para o cálculo, se confirmada a concepção. Apesar deste cálculo da idade à primeira concepção não ser preciso como os atualmente utilizados (p.e. ultrassonografia) e embutir erro, comum a todas as observações, o foco principal da pesquisa era comparar diferentes metodologias para avaliação de precocidade reprodutiva.

Os dados foram analisados inicialmente pelo método da máxima verossimilhança restrita, com a utilização do aplicativo MTDFREML (multiple trait derivative free restricted maximum likelihood; Boldman et al., 1993) para estimação dos componentes de variância e valores genéticos.

O mesmo modelo de análise empregado na estimação pelo método REML foi considerado na análise bayesiana, utilizando-se o conjunto de programas MTGSAM (multiple-trait Gibbs sampler for animal models), desenvolvido por Van Tassel e Van Vleck (1995), para a estimação dos componentes de variância e valores genéticos dos animais.

Inferências acerca da dispersão dos parâmetros foram realizadas a partir das distribuições a posteriori obtidas via amostrador de Gibbs. Para as variâncias genéticas aditiva e residual empregou-se distribuições a priori não informativas.

O programa fortran gibanal versão 2.4 (Van Kaam, 1998) foi utilizado para a análise das cadeias Gibbs, a fim de se calcular o período de burn-in, espaçamento do parâmetro da cadeia e número total de amostras.

O esquema da amostragem Gibbs considerou o tamanho da cadeia de 1005000 , com burn-in de 5000 e interval o de amostragem igual a 1000, resultando em 1000 amostras disponíveis para avaliação das distribuições a posteriori.

Posteriormente os dados foram analisados por modelo linear censurado, sendo que as informações dos animais cujas ocorrências foram vazia, sem notícia e serviu em outra raça, na ocasião da primeira cobertura, foram considerados censurados, tendo como critério de censura a data da segunda cobertura, perfazendo $18,3 \%$ do arquivo de dados. Os acasalamentos que deram origem a macho, fêmea, aborto e natimorto foram tratados como observações não censuradas. Para a realização desta análise procedeu-se a adaptação do programa desenvolvido Por Sorensen et al. (1998), utilizando Fortran 77 em ambiente Windows. Neste caso a amostragem de Gibbs foi realizada a partir do procedimento desenvolvido por Tanner e Wong (1987) e envolveu uma cadeia de 1500000 , com burnin de 10000 e intervalo de amostragem igual a 2500, resultando em 599 estimativas para estudo.

A comparação das metodologias foi realizada a partir dos valores genéticos preditos dos animas para cada método avaliado. Foram obtidas as correlações de ranking entre os valores genéticos dos animais, a partir do programa SAS (1999). Este procedimento visou comparar as metodologias avaliadas, considerando-se as mudanças no posicionamento dos animais nas diferentes classificações e analisar o impacto prático destas metodologias na escolha dos reprodutores.

\section{RESULTADOSEDISCUSSÃO}

A idade média à primeira concepção foi de 60,71 meses, com desvio-padrão de 18,93 meses. Considerando-se o primeiro período médio de gestação de 337,83 dias encontrado por Taveira et al. (2001), a idade à primeira parição ocorreria aproximadamente aos 72 meses, valor inferior aos 76,08 e 82,68 meses, reportados por Valera et al. (2000) e Fuentes et al. (1990), nas raças Lusitana e Árabe, respectivamente. A transformação 
Tabela II. Estimativas obtidas a partir do método da máxima verossimilhança restrita (MTDFREML) e modas, médias, desvios-padrão e intervalos de maior densidade a posteriori com 90\% de probabilidade (HPD 90\%), obtidos por meio de análise bayesiana*, das variâncias genética, ambiente, fenotípica e herdabilidade da idade à primeira concepção em éguas da raça Puro-Sangue Inglês. (Estimates obtained from MTDFREML program, mode, means, standard deviations and a posteriori higher density intervals with a probability of $90 \%$ (HPD $90 \%$ ), obtained by bayesian analysis, for genetic, environment, phenotypic variances and heritability of age at first conception in Thoroughbred mares).

\begin{tabular}{|c|c|c|c|c|c|c|}
\hline \multirow[t]{2}{*}{ Parâmetro } & \multirow[b]{2}{*}{$\begin{array}{l}\text { Estimativa } \\
\text { REML }\end{array}$} & \multicolumn{4}{|c|}{ Análise bayesiana* } & \multirow[b]{2}{*}{$\begin{array}{c}\text { Correlação } \\
\text { serial }\end{array}$} \\
\hline & & Moda & Média & $\begin{array}{l}\text { Desvio } \\
\text { padrão }\end{array}$ & HPD 90\% & \\
\hline Var. genética & 71,33 & 91,74 & 97,08 & 21,58 & $78,55-105,16$ & 0,029 \\
\hline Var. ambiente & 242,11 & 224,33 & 221,49 & 18,91 & $206,66-229,65$ & 0,015 \\
\hline Var. fenotípica & 313,44 & 320,76 & 318,57 & 8,68 & $313,10-323,31$ & 0,021 \\
\hline Herdabilidade & $0,23(0,061)$ & 0,29 & 0,30 & 0,064 & $0,27-0,34$ & 0,064 \\
\hline
\end{tabular}

*Tamanho da cadeia $=1005000$, com burn-in de 5000 e intervalo de amostragem igual a 1000 , resultando em 1000 estimativas. O critério de convergência adotado foi $10^{-9}$.

da idade à primeira concepção em idade à primeira parição foi realizada devido à escassez de literatura acerca da idade à primeira concepção, por tratar-se de uma nova avaliação para características reprodutivas em eqüinos.

Os resultados das estimativas dos componentes de variância obtidas pelo método MTDFREML e pela inferência bayesiana (via MTGSAM) estão apresentados na tabela II.

Pode-se constatar que as estimativas pontuais obtidas da variância genética obtida pelo método MTDFREML apresentam-se inferior à média posteriori obtida pelo método bayesiano. Este resultado concorda, em parte, com os resultados de Wright et al. (2000), os quais, comparando métodos tradicionais e bayesianos em experimentos de criação animal, constataram que a estimativa pontual para o componente de variância genética aditiva obtida pelo método REML era consideravelmente menor que a média a posteriori obtida por inferência bayesiana, mas não para as variâncias ambiente e fenotípica, as quais foram próximas em ambos métodos.

De acordo com Madureira (2003), a inferência bayesiana permite estabelecer regiões de credibilidade ou combinação de parâmetros, as quais são facilmente construídas a partir das amostras obtidas no esquema de amostragem Gibbs. Sendo assim, neste estudo, para a herdabilidade da idade à primeira concepção (figura 1), o intervalo de maior densidade a posteriori, com $90 \%$ de probabilidade (HPD 90\%) foi obtido entre 0,27 e 0,34 (tabela II).

Considerando-se os valores pontuais e a média das estimativas das herdabilidades obtidas pelo método REML e inferência bayesiana, via MTGSAM (tabela II), consideradas de média magnitude, pode esperarse que a IPCON apresente mudança genética razoável mediante seleção massal.

A tabela III apresenta a amplitude dos valores genéticos para a idade à primeira concepção (IPCON) de acordo com as três metodologias de avaliação.

Observou-se que não ocorreu muita variação em relação às amplitudes existente entre os valores genéticos estimados pelo 


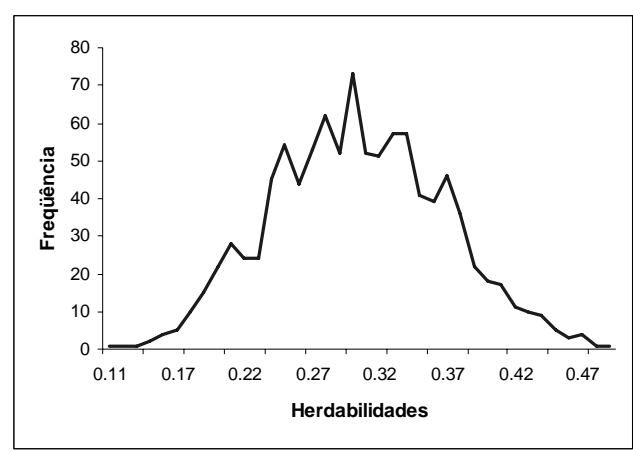

Figura 1. Distribuição da herdabilidade da idade à primeira concepção de animais da raça Puro-Sangue Inglês, obtida por análise bayesiana, via MTGSAM. (Distribution of heritability of age at first conception in Thoroughbred, obtained by bayesian analysis, via MTGSAM).

MTDF e MTGSAM, com grande proximidade entre os valores médios $(-0,16$ e - 0,19), para os dois métodos, respectivamente. Desta maneira, pode-se ressaltar novamente, a grande semelhança entre os resultados obtidos por estas duas metodologias, mesmo com a primeira utilizando a estatística frequentista e a segunda fazendo uso de inferências bayesianas. Em função da escala logarítmica utilizada pelo LMCD na estimação dos valores genéticos dos animais, a comparação com as demais metodologias é inadequada.

De acordo com as figuras 2, 3 e $\mathbf{4}$ que apresentam os valores genéticos médios da
Tabela III. Amplitude dos valores genéticos para a idade à primeira concepção (IPCON) de éguas da raça Puro-Sangue Inglês, em função das metodologias de avaliação. (Amplitude of breeding values for age at first conception (AFCON) for Thoroughbred mares, in function of evaluation methodologies).

\begin{tabular}{lccc}
\hline Metodologia & Mínimo & Média & Máximo \\
\hline MTDF (meses) & $-13,68$ & $-0,16$ & 32,61 \\
MTGSAM (meses) & $-16,66$ & $-0,19$ & 43,19 \\
LMCD (escala log) & $-0,0955$ & $-0,0013$ & 0,1186 \\
\hline
\end{tabular}

idade à primeira concepção em éguas da raça Puro-Sangue Inglês em função do ano de nascimento, de acordo com as três metodologias avaliadas, observa-se que está ocorrendo aumento de 0,0175 meses/ ano ou 1,75 meses a cada 10 anos, (MTDFREML), representando aumento anual de $0,028 \%$ em relação a média da característica, $0,4 \%$ em relação ao desvio padrão genético e $0,098 \%$ em relação ao desvio padrão fenotípico. Considerandose a análise bayesiana por meio do programa MTGSAM, observou-se aumento de 0,0249 meses / ano ou 2,49 meses a cada 10 anos (MTGSAM), representando aumento anual de $0,04 \%$ em relação à média da característica, $0,25 \%$ em relação ao desvio padrão genético e $0,14 \%$ em relação ao desvio padrão fenotípico. A tendência no aumento $(0,0001$ et al., 1999 e Pereira et al., 2004), pois não

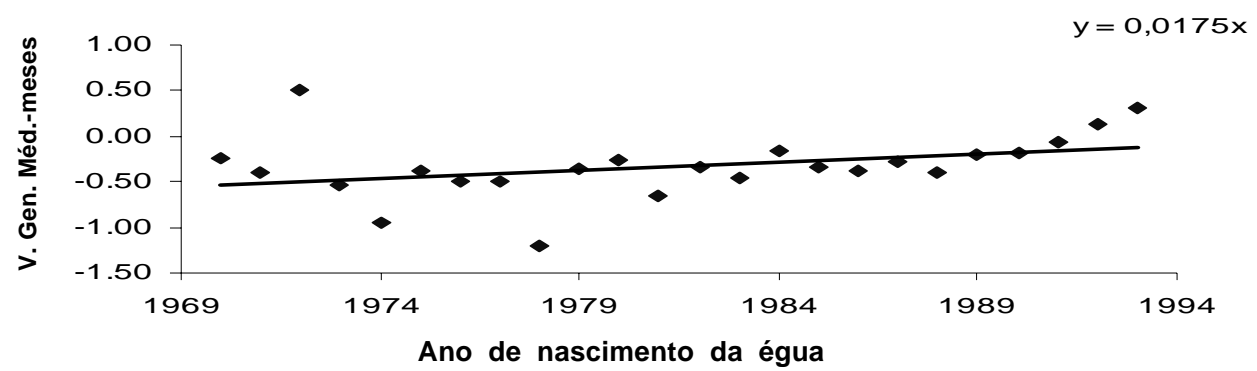

Figura 2. Tendência genética da idade à primeira concepção em éguas da raça Puro-Sangue Inglês (MTDF). (Genetic trend for age at first conception in Thoroughbred mares (MTDF)). 


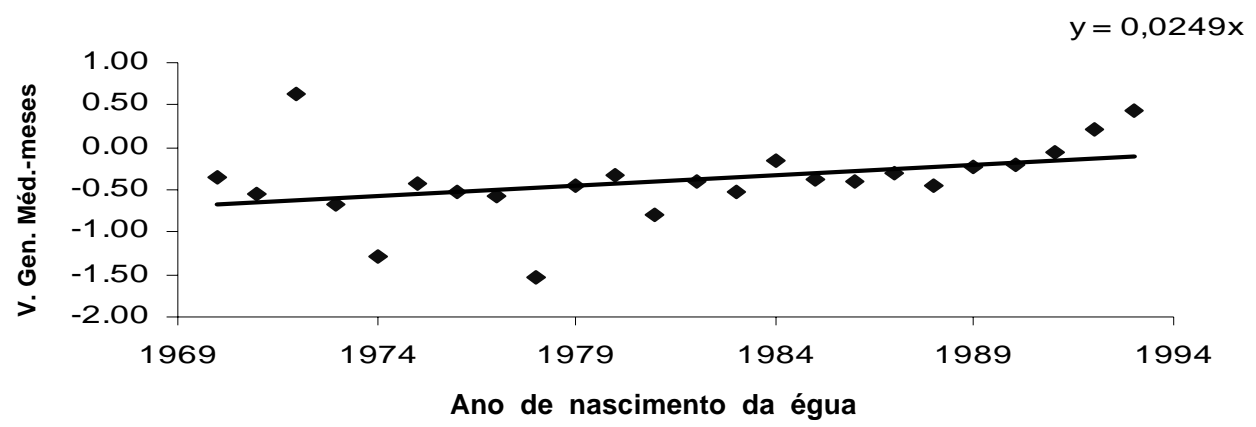

Figura 3. Tendência genética da idade à primeira concepção em éguas da raça Puro-Sangue Inglês (MTGSAM). (Genetic tendency for age at first conception in Thoroughbred mares (MTGSAM)).

em escala log) da idade à primeira concepçao, também foi observada quando utilizado o modelo linear com dados censurados (LMCD), apresentando aumento anual de $0,10 \%$ em relação ao desvio padrão genético e $0,03 \%$ em relação ao desvio padrão fenotípico. Esta tendência pode ser, em parte, reflexo da pouca atenção dada às características de precocidade sexual no processo de seleção dos animais, o que, em última análise, contribuiu para a baixa eficiência reprodutiva da criação de animais da raça Puro-Sangue Inglês no país.

Observa-se que o mérito genético aditivo médio dos animais apresentou maior dispersão em relação aos valores estimados no período entre 1969 a 1980. Posteriormente, quando registrou-se maior número de animais por ano, o mérito genético médio estimado foi mais próximo que o observado, com pequenas flutuações em um ou outro sentido, até 1992, ano a partir do qual a tendência passou a ser desfavorável.

Os valores mínimos, máximos, médias, modas, medianas, HPD 90\% e correlação serial dos componentes de variância e herdabilidade em escala logarítmica obtidos por análise bayesiana, via LMCD estão apresentados na tabela IV.

Ressalva-se, no entanto, que a interpretação da herdabilidade na escala logarítmica tem sido alvo de discussão (Korsgaard

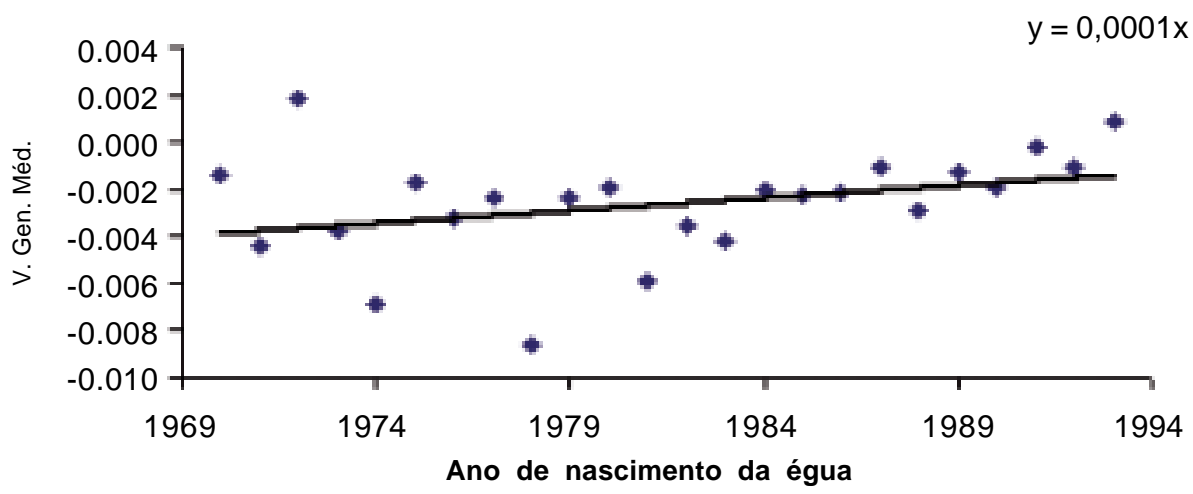

Figura 4. Tendência genética da idade à primeira concepção em éguas da raça Puro-Sangue Inglês, em escala logarítmica (LMCD). (Genetic trend for age at first conception in Thoroughbred mares, on logarithmical scale (LMCD)). 
Tabela IV. Estimativa de parâmetros para a idade à primeira concepção em éguas da raça Puro-Sangue Inglês obtidos por modelo linear censurado via amostragem de Gibbs ${ }^{a}$. (Parameter estimate for age at first conception in Thoroughbred mares obtained by a censored linear model via Gibbs ${ }^{a}$ sampling).

\begin{tabular}{lccc}
\hline Fator & $\mathrm{h}_{(\mathrm{log})}^{2}$ & $\sigma_{\mathrm{a}(\mathrm{log})}^{2}$ & $\sigma_{\text {e(log) }}^{2}$ \\
\hline Média & 0,0926 & 0,0084 & 0,0816 \\
Moda & 0,0723 & 0,007 & 0,0830 \\
Mediana & 0,0835 & 0,008 & 0,0820 \\
Mínimo & 0,004 & 0,001 & 0,066 \\
Máximo & 0,304 & 0,29 & 0,095 \\
HPD 90\% & 0,041 e 0,099 & $0,007-0,017$ & $0,069-0,082$ \\
Correlação serial & 0,028 & 0,042 & 0,021 \\
\hline
\end{tabular}

aTamanho da cadeia = 1500 000, com burn-in de 10000 e intervalo de amostragem igual a 2500, resultando em 599 estimativas.

$\mathrm{h}^{2}(\mathrm{log})=$ herdabilidade na escala logarítmica.

$\sigma^{2}{ }_{a(\log )}^{(\log )}=$ variância genética na escala logarítmica.

$\sigma^{2}{ }_{e(l o g)}=$ variância ambiente na escala logarítmica.

se apresenta de maneira direta como no caso dos métodos REML e inferência bayesiana, via MTGSAM, dificultando a comparação com estes dois últimos métodos. Entretanto, correlações de ranking podem ser utilizadas para comparação das metodologias utilizadas com base no posicionamento dos animais pelo valor genético, obtido dentro de cada metodologia utilizada.

As correlações obtidas entre as posições de classificação ranking dos animais avaliados pelo valor genético direto quando comparados os modelos REML e análise Bayesiana via MTGSAM, foram altas e positivas (tabela V).

Tabela $\boldsymbol{V}$. Correlações de ranking entre os valores genéticos dos animais para idade à primeira concepção de éguas da raça Puro-Sangue Inglês, pelo método da máxima verossimilhança restrita e análise bayesiana por meio dos programas MTGSAM e LMCD. (Ranking correlation between animals' breeding values for age at first conception of Thoroughbred mares, by restricted maximum likelihood method and bayesian analysis by MTGSAM and LMCD programs).

\begin{tabular}{|c|c|c|c|c|}
\hline \multirow{2}{*}{ Classe de animais } & \multirow[t]{2}{*}{$\mathrm{N}$} & \multicolumn{3}{|c|}{ Correlação de ranking } \\
\hline & & LMCD-MTDF & LMCD-MTGSAM & MTDF-MTGSAM \\
\hline Todos os animais do pedigree & 6713 & 0,91 & 0,90 & 0,99 \\
\hline $10 \%$ melhores animais & 671 & 0,62 & 0,57 & 0,96 \\
\hline $5 \%$ melhores animais & 335 & 0,59 & 0,54 & 0,95 \\
\hline $1 \%$ melhores animais & 67 & 0,58 & 0,52 & 0,95 \\
\hline Todos os garanhões & 656 & 0,91 & 0,90 & 0,99 \\
\hline $10 \%$ melhores garanhões & 65 & 0,58 & 0,55 & 0,97 \\
\hline $5 \%$ melhores garanhões & 32 & 0,52 & 0,48 & 0,96 \\
\hline $1 \%$ melhores garanhões & 6 & 0,65 & 0,69 & 0,93 \\
\hline
\end{tabular}


Tabela VI. Posicionamento dos 10 melhores animais no arquivo geral em função das metodologias de análise. (Ten top ranking animals in the general files in function of analysis methodologies).

\begin{tabular}{|c|c|c|c|}
\hline Animal & LMCD & MTDF & MTGSAM \\
\hline$A$ & 10 & 3응 & 3음 \\
\hline$B$ & $2^{\circ}$ & 160 & 180 \\
\hline C & $3^{\circ}$ & $19 \circ$ & $36^{\circ}$ \\
\hline$D$ & $4^{\circ}$ & 10 & 10 \\
\hline$E$ & 50 & 35으 & 290 \\
\hline$F$ & $6^{\circ}$ & $8^{\circ}$ & $6^{\circ}$ \\
\hline $\mathrm{G}$ & $7^{0}$ & $11^{\circ}$ & $10^{\circ}$ \\
\hline $\mathrm{H}$ & $8^{\circ}$ & $21^{\circ}$ & $24^{\circ}$ \\
\hline I & 9o & $128^{\circ}$ & $197^{\circ}$ \\
\hline$J$ & $10^{\circ}$ & $38^{\circ}$ & 52 \\
\hline
\end{tabular}

Entretanto, quando estas mesmas metodologias foram contrastadas com a análise Bayesiana, via LMCD, apresentaram correlação de ranking de menor magnitude, trazendo reflexos na classificação dos animais de acordo com a metodologia utilizada, como pode ser observado nos 10 melhores animais classificados de acordo com a análise Bayesiana, via LMCD (tabela VI).

Com base na tabela acima, nota-se que para o efeito genético direto dos 10 melhores animais classificados, somente três seriam selecionados pelas três metodologias de análise utilizadas, sendo que a mudança mais expressiva ocorreu com o animal I, que na análise bayesiana, via LMCD, encontrase na $9^{\text {a }}$ posição, e no entanto ocupa a $128^{\text {a }}$ e $197^{\text {a }}$ posição pelo método do REML e análise bayesiana via MTGSAM, respectivamente. Pode-se observar que este animal tratava-se de um garanhão que possuía filhas com informação de censura, o que possivelmente, pode ter causado a melhoria no posicionamento de seu ranking no LMCD, que leva em consideração tais informações.

Pode-se observar bastante semelhança entre os rankings considerando-se método da máxima verossimilhança restrita e análise bayesiana por meio do MTGSAM. Quando observado somente os garanhões, esta semelhança ainda permanece, inclusive quando se analisa os $10 \%, 5 \%$ e $1 \%$, indicando bastante similaridade entre estas duas metodologias de análise (tabela V).

Analisando-se os rankings entre a análise bayesiana via LMCD e os métodos da máxima verossimilhança restrita e análise bayesiana via MTGSAM, percebe-se, considerando todos os animais do pedigree, diminuição na semelhança entre os rankings $(0,91$ e 0,90$)$ quando comparados com o ranking entre os dois últimos $(0,99)$. Verifica-se que quanto maior a pressão de seleção considerada, menor é a correlação entre as metodologias. Assim, dentre os $10 \%$ melhores animais classificados pelo LMCD, a correlação diminui para 0,62 e 0,57, para rankings com o REML e MTGSAM, respectivamente. Estes valores decrescem ainda mais (tabela IV), quando aumenta-se a pressão de seleção (5 e 1\%), chegando ao mínimo de 0,52 entre a análise bayesiana via MTGSAM e LMCD. Valor semelhante foi observado entre LMCD e MTDF para os 5\% melhores garanhões. Correlações superiores as anteriores observadas entre o LMCD e as outras metodologias, quando avaliouse $1 \%$ dos melhores garanhões, podem ser explicadas pela baixa quantidade de animais envolvida, já que nesta situação, a correlação de Sperman torna-se bastante sensível a pequenas alterações de ranqueamento.

De modo geral, estes resultados concordam com os achados de Vollema e Groen (1998), em gado de leite, os quais observaram que a inclusão de informações censuradas na análise, diminuia o valor das correlações entre o valor genético dos animais, fornecendo um ranking de animais consideravelmente diferente. Assim, considerando-se que a exclusão de dados censurados em análises de características como IPCON provoca séria distorção das inferências (Sorensen et al., 1998), observa-se que haveria considerável divergência entre 


\section{IDADE À PRIMEIRA CONCEPÇÃO EM ÉGUAS PURO-SANGUE INGLÊS}

Tabela VII. Porcentagem de coincidentes de acordo com o método utilizado e fração selecionada no arquivo de pedigree. (Percentage of coincident according to the used method and selected fraction in the pedigree files).

Classe de animais

Porcentagem de coincidentes (\%)

LMCD-MTDF LMCD-MTGSAM MTDF-TGSAM

Todos os animais do pedigree

$10 \%$ melhores animais

$5 \%$ melhores animais

73,77

71,34

$1 \%$ melhores animais

Todos os garanhões

$10 \%$ melhores garanhões

$5 \%$ melhores garanhões

$1 \%$ melhores garanhões
53,73

75,38

71,87

50,00
78,09

68,35

53,73

75,38

65,62

50,00
93,44

93,43

88,05

92,30

90,62

83,33
LMCD e as outras metodologias no momento da escolha dos animais geneticamente superiores, principalmente em garanhões, onde há maior pressão de seleção.

Na tabela VII é apresentada uma análise comparativa da coincidência (representa o número de animais selecionados por ambos os métodos) na classificação dos $10 \%, 5 \%$ e $1 \%$ melhores animais no arquivo de pedigree e arquivo de machos, em função das metodologias de análise. Assim como na correlação de ranking observa-se que quanto maior a pressão de seleção, menor a coincidência entre os animais selecionados.

Este comportamento acentua-se ainda mais quando avaliado apenas os garanhões, chegando apenas a $50 \%$ de coincidência quando comparado o LMCD com as demais metodologias. Entretanto, este valor deve ser visto com ressalva devido ao pequeno número de animais nesta categoria.

Quando avaliado 1\% dos melhores animais no arquivo de pedigree, onde possivelmente ocorreria a seleção dos animais para reprodução, observa-se que $46,27 \%$ dos animais selecionados pelo LMCD seriam descartados pelas demais metodologias. Admitindo-se que o modelo linear censurado é mais apropriado para se avaliar características com informações censuradas (Tempelman, 1997) como IPCON, a discrepância entre os animais selecionados por este método e os outros dois é considerável. Isto pode significar que as metodologias normalmente utilizadas podem não identificar eficientemente os animais geneticamente superiores naquela característica. Por outro lado a alta porcentagem de coincidentes entre MTDF e MTGSAM indica que menos que $17 \%$ (1\% dos melhores garanhões) dos selecionados por um método seriam descartados pelo outro.

\section{CONCLUSÕES}

As estimativas de herdabilidade encontradas neste estudo sugerem que a idade à primeira concepção pode ser utilizada como critério de seleção em criações de PuroSangue Inglês que busquem maior precocidade sexual.

A tendência genética de aumento na IPCON deve-se, em parte, a sua não inclusão em programas de melhoramento da raça, a qual se baseia, principalmente, no desempenho em pista dos animais.

A utilização de modelos que consideram dados censurados em estudos de características reprodutivas em eqüinos é uma opção de análise que pode trazer benefícios no melhoramento destas características nesta espécie. Apesar da implementação da 


\section{IDADE À PRIMEIRA CONCEPÇÃO EM ÉGUAS PURO-SANGUE INGLÊS}

análise envolvendo o modelo linear censurado apresentar-se mais difícil que os outros dois métodos utilizados, mostrou-se adequada para o estudo de características censuradas como a idade à primeira concepção.

Embora o sucesso na indústria do PuroSangue Inglês seja medido pelo desempenho

\section{BIBLIOGRAFIA}

Boldman, K., Van Vleck, L.D. e Kriese, L.A. 1993. A manual for use of MTDFREML - a set of programs to obtain estimates of variances and covariances. USDA-ARS. USA.

Blasco, A. 2001. The bayesian controversy in animal breeding. J. Anin. Sci., 79: 2023-2046.

Cilek, S. 2009. The survey of reproductive success in Arabian horse breeding from 1976-2007 at Anadolu State Farm in Turkey. J. Anin. Vet. Adv., 8: 389-396.

Cunningham, E.P. 1991. The genetics of Thoroughbred horses. Sci. Amer., 5: 92-98.

Fuentes, F., Gonzalo, C., Herrera, M., Quiles, A. y Hevia, M. 1990. Parámetros reproductivos del caballo arabe. ITEA., 86: 172-177.

Gianola, D. 2000. Statistics in animal breeding. J. Amer. Stat. Ass., 95: 296-299.

Korsgaard, I.R., Andersen, A.H. and Jensen, J. 1999. Discussion of heritability of survival traits. Int. Bull., 21: 31-35.

Madureira, A.P. 2003. Comparação de modelos lineares mistos gaussianos e robustos em características de crescimento de bovinos de corte. (Tese de doutorado). FMVZ. Botucatu. 89 pp.

Pereira, E. 2004. Diferentes abordagens para o estudo da precocidade sexual de fêmeas Nelore. (Tese de doutorado). FMVZ. Botucatu. 62 pp.

SAS. 1999. User's guide: basics and statistics. SAS Inst. Inc. Cary. USA. 956 pp.

Sorensen, D.A., Gianola, D. and Korsgaard, I.R. 1998. Bayesian mixed-effects model analysis of a censored normal distribution with animal breeding applications. Acta Agric. Scand. Sect. dos animais em pista, é importante que as características reprodutivas também sejam contempladas em programas de seleção, uma vez que possibilitariam maior intensidade seletiva e ganhos genéticos maielevados naquele desempenho.

\section{A. Animal Sci., 48: 222-229.}

Tanner, M.A. and Wong, W.H. 1987. The calculation of posterior distributions by data augmentation. J. Anim. Stat. Assoc., 82: 528-540.

Taveira, R.Z., Carvalho, D. e Oliveira, P.S. 2001. Avaliação de características de desempenho em produtos Nelore $X$ Limousin. $38^{\circ}$ Congresso Brasileiro de Zootecnia. Piracicaba. SP. Brasil. $425 \mathrm{pp}$.

Templeman, R.J. 1997. Generalized linear mixed model in dairy cattle breeding. J. Dairy Sci., 81: 1428-1444.

Valera, M., Esteves, M.M. and Molina, A. 2000. The Lusitano native Thoroughbred: A genetics study of the important reproductive parameters in plans for conservation and improvement. Arch. Zootec., 49: 147-156.

Van Kaam, J.B.C.H.M. 1998. www.student.wau.nl/ janthijs/breedingsite/edagibanal.html. (20/05/ 08).

Van Tassel, C.P. and Van Vleck, L.D. 1995. A manual for use of MTGSAM. A set of Fortram programs to apply Gibbs sampling to animal models for variance component estimation. [DRAFT]. Department of Agriculture. Agricultural Research Service. USA.

Vollema, A.R. and Groen, A.F. A. 1998. Comparison of breeding value predictors for longevity using a linear model and survival analysis. J. Dairy Sci., 81: 3315-3320.

Wright, D.R., Stern, H.S. and Berger, P.J. 2000. Comparing traditional and bayesian analyses of selection experiments in animal breeding. $J$. Agric. Biol. Environ. Stat., 5: 240-256. 